TRANSACTIONS OF THE

AMERICAN MATHEMATICAL SOCIETY

Volume 290, Number 1, July 1985

\title{
ON THE BOUNDARY BEHAVIOUR OF GENERALIZED POISSON INTEGRALS ON SYMMETRIC SPACES
}

\author{
BY
}

HENRIK SCHLICHTKRULL ${ }^{1}$

\begin{abstract}
On a Riemannian symmetric space $X$ of the noncompact type we introduce a generalized Poisson transformation from functions on the minimal boundary to functions on the maximal compactification whose restrictions to $X$ are eigenfunctions of the invariant differential operators. Some continuity- and "Fatou"-theorems are proved.
\end{abstract}

Introduction. Let $X=G / K$ be a Riemannian symmetric space of the noncompact type. By a theorem of Kashiwara et al. [5] every joint eigenfunction $\phi$ of the invariant differential operators on $X$ can be represented as a generalized Poisson integral of a hyperfunction $\varphi$ on the minimal boundary $K / M$, and, for "generic" eigenvalues, $\varphi$ is the "boundary value" of $\phi$ in a certain abstract sense. When $\varphi$ is a continuous function, $\varphi$ is the boundary value of $\phi$ in a much more concrete way: $\varphi$ is the limit along geodesics of the ratio $\phi / \phi_{\lambda}$ of $\phi$ with the spherical function $\phi_{\lambda}$ with the same eigenvalues as $\phi$. This result holds under the condition on the eigenvalues that $\operatorname{Re} \lambda$ lies in the positive open Weyl chamber, and it is due to Helgason [1, p. 130]. It was generalized to $L^{\infty}$-functions $\varphi$ on $K / M$ with pointwise almost everywhere "admissible" convergence by Michelson [12, Theorem 2.2] and to $L^{1}$-functions $\varphi$ on $K / M$ with pointwise almost everywhere "restricted admissible" convergence by Sjögren [15, Theorem 2]. For $\phi$ harmonic (which is the same as $\lambda=\rho$ ) these "Fatou-theorems" had been proved by Helgason, Korányi, Knapp, Williamson, Lindahl and Stein $[4,6,7,8,11,16]$.

In this note we generalize these results of Helgason, Michelson and Sjögren to take into consideration the full boundary of $X$. In the maximal Satake-Furstenberg compactification $\bar{X}$ of $X, K / M$ is only part of the boundary of $X$ (unless rank $G / K=1)$-it is the unique compact $G$-orbit. The other $G$-orbits in the boundary can be identified with homogeneous spaces of the form $G / B$, where $B$ is given by $B=(M \cap K) A N$ for a parabolic subgroup $P=M A N$ of $G$. For each such $G$-orbit we define a generalized "partial" Poisson transformation from functions on $K / M$ to sections of line bundles over $G / B$. We will prove that if $\varphi$ is continuous, the Poisson

Received by the editors March 21, 1984 and, in revised form, August 15, 1984.

1980 Mathematics Subject Classification. Primary 43A85; Secondary 53C35.

Key words and phrases. Poisson integral, symmetric space, compactification, boundary, Fatou theorem.

${ }^{1}$ Research supported by The Danish Natural Science Research Council. 
transform of $\varphi$ on $G / B$ is the limit of $\phi$ on $X$ (after a normalization analogous to the division with $\phi_{\lambda}$ above). (This is done in $\S_{2}$.) Moreover, for $\varphi \in L^{p}(1 \leqslant p \leqslant \infty)$, we prove convergence almost everywhere (in $\S 3$ ). In the harmonic case $(\lambda=\rho)$ these results for the full boundary have been proved by Koranyi $[9,10]$ and Stein $[16]$, and our method of proof is in fact by developing generalizations of their proofs. For simplicity we confine ourselves to the maximal compactification, leaving the further generalization to the other Satake-Furstenberg compactifications (cf. Koranyi [9, Lemma 1.2]) to the imagination of the reader.

Finally we mention that the theorem of Kashiwara et al. mentioned above, valid for hyperfunctions $\varphi$, can also be generalized to this setting with the full boundary of $X$ (see [14, Chapter 6]).

1. The generalized Poisson transformations. Let $G$ be a connected real noncompact semisimple Lie group with finite center, $G=K A N$ an Iwasawa decomposition of $G$, a the Lie algebra of $A$ and $\kappa: G \rightarrow K, H: G \rightarrow$ a the corresponding projections. Let $\theta$ denote the Cartan involution, let $\bar{N}=\theta(N)$ and let $M$ be the centralizer of $a$ in $K$. Let $a^{*}$ (respectively $\mathfrak{a}_{c}^{*}$ ) be the real (complex) dual of $a, \Sigma \subset a^{*}$ the system of restricted roots, $\Sigma^{+}$the set of positive roots in $\Sigma, \rho$ half the sum of the roots in $\Sigma^{+}$ with multiplicities and $a_{+}^{*}$ the Weyl chamber in $a^{*}$ consisting of those $\lambda \in a^{*}$ such that $\langle\lambda, \alpha\rangle>0$ for all $\alpha \in \Sigma^{+}$, where $\langle$,$\rangle denotes the Killing form. We write$ $\lambda \in a_{c}^{*}$ as $\lambda=\operatorname{Re} \lambda+\sqrt{-1} \operatorname{Im} \lambda$, where $\operatorname{Re} \lambda, \operatorname{Im} \lambda \in a^{*}$. Let $n=\operatorname{dim}$ a, let $\Delta=$ $\left\{\alpha_{1}, \ldots, \alpha_{n}\right\}$ be the set of simple roots for $\Sigma^{+}$and let $\left(H_{1}, \ldots, H_{n}\right)$ be the basis for a dual to $\Delta$. For $a \in A$ and $\nu \in a_{c}^{*}$ let $a^{\nu}=\exp \langle\nu, H(a)\rangle$.

For each subset $F \subset \Delta$ let $\mathfrak{a}_{F}$ be its annihilator in $\mathfrak{a}$, and let $\mathfrak{a}_{F}^{+}$consist of those $H \in a_{F}$ for which $\alpha(H)>0$ for all $\alpha \in \Delta \backslash F$. Let $A_{F}=\exp a_{F}$ and let $P_{F}=$ $M_{F} A_{F} N_{F}$ be the corresponding parabolic subgroup with the indicated Langlands decomposition and $N_{F} \subset N$. Let $B_{F}$ be the subgroup $B_{F}=\left(M_{F} \cap K\right) A_{F} N_{F}$ of $P_{F}$, and let $\bar{N}_{F}=\theta\left(N_{F}\right), N(F)=M_{F} \cap N$ and $\bar{N}(F)=\theta(N(F))$.

Let $f$ be an integrable function on $K / M$. For each $F \subset \Delta$ and $\lambda \in \mathfrak{a}_{c}^{*}$ we define the ( generalized) partial Poisson integral of $f$ as the function $\mathscr{P}_{\lambda}^{F} f$ on $G$ given by

$$
\mathscr{P}_{\lambda}^{F} f(g)=\int_{M_{F} \cap K} f(\kappa(g k)) e^{\langle\lambda-\rho . H(g k)\rangle} d k
$$

(this is well defined for a.a. $g \in G$ ). Let $f_{\lambda}$ be the function

$$
f_{\lambda}(g)=f(\kappa(g)) e^{\langle\lambda-\rho, H(g)\rangle}
$$

on $G$. Then

$$
\mathscr{P}_{\lambda}^{F} f(g)=\int_{M_{F} \cap K} f_{\lambda}(g k) d k
$$

The transformation $\mathscr{P}_{\lambda}=\mathscr{P}_{\lambda}^{\Delta}$ is the (generalized) Poisson transformation which takes functions on $K / M$ to eigenfunctions on $G / K$ for the invariant differential operators. When $\lambda=\rho$, these Poisson integrals on $G / K$ are harmonic functions.

It is easily seen that

$$
\mathscr{P}_{\lambda}^{F} f(\text { gman })=a^{\lambda-\rho} \mathscr{P}{ }_{\lambda}^{F}(g)
$$

for all $g \in G, m \in M_{F} \cap K, a \in A_{F}$ and $n \in N_{F}$. 
Let 1 denote the constant function with value 1 on $K / M$ and let $\phi_{\lambda}^{F}=\mathscr{P}_{\lambda}^{F} \mathbf{1}$. Then $\phi_{\lambda}^{\Delta}$ is the spherical function

$$
\phi_{\lambda}(g)=\int_{K} e^{\langle\lambda-\rho, H(g k)\rangle} d k
$$

on $G$. We define the normalized partial Poisson integral of $f$ by

$$
\mathfrak{p}_{\lambda}^{F} f(g)=\mathscr{P}_{\lambda}^{F} f(g) / \phi_{\lambda}^{F}(g)
$$

for $g \in\left\{g^{\prime} \in G \mid \phi_{\lambda}^{F}\left(g^{\prime}\right) \neq 0\right\}$. Then $\mathfrak{p}_{\lambda}^{F} f(g b)=\mathfrak{p}_{\lambda}^{F} f(g)$ for $b \in B_{F}$. Notice that if $\lambda \in a^{*}$, then $\phi_{\lambda}^{F}(g) \neq 0$ for all $g \in G$.

If we define an action of $G$ on functions $f$ on $K / M$ by left translation of the corresponding functions $f_{\lambda}$, it is obvious that $\mathscr{P}_{\lambda}^{F}$ is a $G$-map. Clearly $\mathfrak{p}_{\lambda}^{F}$ is a $K$-map.

Let $\bar{X}$ be the maximal Satake-Furstenberg compactification of $X$. The space $\bar{X}$ can be constructed as follows (cf. Oshima [13], or [14, Chapter 4]). Let $\mathbf{R}_{+}^{n}=\left[0, \infty\left[{ }^{n}\right.\right.$ and for $t \in \mathbf{R}_{+}^{n}$ let $F_{t}=\left\{\alpha_{j} \in \Delta \mid t_{j} \neq 0\right\}$ and $a_{t}=\exp \left(-\sum_{\alpha_{j} \in F_{t}}\left(\log t_{j}\right) H_{j}\right)$ (and $a_{0}=$ $e)$. Define an equivalence relation $\sim$ on $G \times \mathbf{R}_{+}^{n}$ by $(g, t) \sim\left(g^{\prime}, t^{\prime}\right)$ if and only if $F_{t}=F_{t^{\prime}}$ and $g a_{t} \in g^{\prime} a_{t^{\prime}} B_{F_{i}}$. Then $\bar{X}=G \times \mathbf{R}_{+}^{n} / \sim$ as a topological space. Let $\pi$ : $G \times \mathbf{R}_{+}^{n} \rightarrow \bar{X}$ be the projection. From the action of $G$ on the first factor of $G \times \mathbf{R}_{+}^{n}$ the space $\bar{X}$ inherits a natural $G$-action, and the orbital decomposition is easily seen to be given by

$$
\bar{X}=\bigcup_{F \subset \Delta} G / B_{F}
$$

(disjoint union), each space $G / B_{F}$ being identified with the subset $\pi\left(\left\{(g, t) \mid F_{t}=F\right\}\right)$ of $\bar{X}$. In particular, $X=G / K$ is identified with $\pi\left(\left\{(g, t) \mid t_{j}>0, \forall_{j}\right\}\right)$ and $K / M=$ $G / P_{\varnothing}$ with $\pi(G \times\{0\})$.

Let $\bar{X}_{\lambda}=\left\{x \in \bar{X} \mid x=g B_{F} \in G / B_{F}\right.$ and $\left.\phi_{\lambda}^{F}(g) \neq 0\right\}$. If $\lambda$ is real valued on $a$, then $\bar{X}_{\lambda}=\bar{X}$. We define the (normalized) Poisson transform on $\bar{X}$ of $f$ as the function $\overline{\mathfrak{p}}_{\lambda} f$ on $\bar{X}_{\lambda}$ whose restriction to $G / B_{F}$ is $\mathfrak{p}_{\lambda}^{F} f$ for all $F \subset \Delta$. In particular, $\overline{\mathfrak{p}}_{\lambda} f(x)=f(x)$ for $x \in K / M \subset \bar{X}$.

2. Continuity theorems. Let $F \subset \Delta$ and $\lambda \in a_{c}^{*}$. Then it is well known that the integral

$$
c_{\lambda}^{F}=\int_{\bar{N}_{F}} e^{\langle-\lambda-\rho, H(\bar{n})\rangle} d \bar{n}
$$

is absolutely convergent and nonzero if $\operatorname{Re} \lambda \in \mathfrak{a}_{+}^{*}$. In the following we use the notation $a \underset{F}{\rightarrow} \infty$ for $a \in A_{F}$ and $a^{\alpha} \rightarrow \infty$ for all $\alpha \in \Delta \backslash F$. For any manifold $S$ let $C(S)$ denote the space of continuous functions on $S$.

THEOREM 1. Let $\lambda \in a_{c}^{*}$ be such that $\operatorname{Re} \lambda \in a_{+}^{*}$, and let $f \in C(K / M)$. Then

$$
a^{\rho-\lambda} \mathscr{P}_{\lambda} f(g a) \rightarrow c_{\lambda}^{F} \mathscr{P}_{\lambda}^{F} f(g)
$$

as $a \underset{F}{\rightarrow} \infty$ for all $g \in G$. The convergence is uniform in $g$ on compact sets. 
Proof. By a well-known integration formula (see [17, Lemma 4.2.14b]) we have

$$
\mathscr{P} f(g)=\int_{M_{F} \cap K} \int_{\bar{N}_{F}} f_{\lambda}(g k \bar{n}) e^{\langle-\lambda-\rho, H(\bar{n})\rangle} d \bar{n} d k
$$

for $g \in G$, and hence for $a \in A_{F}$

$$
a^{\rho-\lambda} \mathscr{P} f(g a)=\int_{M_{F} \cap K} \int_{\bar{N}_{F}} f_{\lambda}\left(g k a \bar{n} a^{-1}\right) e^{\langle-\lambda-\rho, H(\bar{n})\rangle} d \bar{n} d k .
$$

Since $a \bar{n} a^{-1} \rightarrow e$ for $a \rightarrow \vec{F} \infty$, an interchange of the order of going to the limit and integrating proves the theorem. The interchange is justified by the dominated convergence theorem by the argument of Helgason [1, p. 130], which in fact is Theorem 1 for $F=\varnothing$.

From Theorem 1 we get the following generalization. Let $F \subset E \subset \Delta$. Then the integral

$$
c_{\lambda}^{F}(E)=\int_{\bar{N}_{F} \cap \bar{N}(E)} e^{\langle-\lambda-\rho, H(\bar{n})\rangle} d \bar{n}
$$

is absolutely convergent and nonzero if $\operatorname{Re} \lambda \in \mathfrak{a}_{+}^{*}$. This follows from (2.1) applied to $M_{E}$ instead of $G$ (in fact $\left.c_{\lambda}^{F}(E)=\left(c_{\lambda}^{E}\right)^{-1} c_{\lambda}^{F}\right)$.

Corollary 1. Let $\lambda \in a_{c}^{*}$ be such that $\operatorname{Re} \lambda \in a_{+}^{*}$ and let $f \in C(K / M)$. Then for all $g \in G$

$$
a^{\rho-\lambda} \mathscr{P}_{\lambda}^{E} f(g a) \rightarrow c_{\lambda}^{F}(E) \mathscr{P}_{\lambda}^{F} f(g)
$$

when $a \in A_{F}$ and $a \rightarrow \infty$ in the sense that $a^{\alpha} \rightarrow \infty$ for all $\alpha \in E \backslash F$.

Proof. By the $G$-invariance of $\mathscr{P}_{\lambda}^{F}$ and $\mathscr{P}_{\lambda}^{E}$ we may assume $g=e$. Then we are actually only considering the restriction of $\mathscr{P}_{\lambda}^{E} f$ to $M_{E}$, and the result follows from Theorem 1 applied to $M_{E}$ instead of $G$.

We now consider the normalized Poisson transform $\overline{\mathfrak{p}}_{\lambda} f$ on $\bar{X}_{\lambda}$. First we need a result about $\bar{X}_{\lambda}$.

Lemma 1. Let $\lambda \in \mathfrak{a}_{c}^{*}$ and assume $\operatorname{Re} \lambda \in \mathfrak{a}_{+}^{*}$. Then $\bar{X}_{\lambda}$ is open.

Proof. Fix $F \subset \Delta$ and let $x \in \bar{X}_{\lambda} \cap G / B_{F}$. Let $g_{0} \in G$ and $t_{0} \in \mathbf{R}_{+}^{n}$ be such that $x=\pi\left(g_{0}, t_{0}\right)$. Then $t_{0 j}=0$ if $\alpha_{j} \notin F$. By choosing $g_{0}$ appropriately we obtain also $t_{0 j}=1$ if $\alpha_{j} \in F$. Let $\Omega$ be a compact neighborhood of $g_{0}$ in $G$ such that $\phi_{\lambda}^{F}(g) \neq 0$ for all $g \in \Omega$. For each $s>0$ let

$$
R_{s}=\left\{t \in \mathbf{R}_{+}^{n} \mid t_{j}=1 \text { if } \alpha_{j} \in F \text { and } t_{j}<s \text { otherwise }\right\}
$$

and let $\Omega_{s}=\pi\left(\Omega \times R_{s}\right)$. Then $\Omega_{s}$ is a neighborhood of $x$ in $\bar{X}$.

Let $E \subset \Delta$ with $F \subset E$, and $R_{s}(E)=\left\{t \in \Omega_{s} \mid F_{t}=E\right\}$. Then $\Omega_{s} \cap G / B_{E}=$ $\pi\left(\Omega \times R_{s}(E)\right)$. From Corollary 1 it follows that

$$
a_{t}^{\rho-\lambda} \phi_{\lambda}^{E}\left(g a_{t}\right) \rightarrow c_{\lambda}^{F}(E) \phi_{\lambda}^{F}(g)
$$

as $t \in R_{s}(E)$ and $s \rightarrow 0$. The convergence is uniform in $g \in \Omega$. Since $c_{\lambda}^{F}(E) \neq 0$ it follows that $\phi_{\lambda}^{E}\left(g a_{t}\right) \neq 0$ for all $g \in \Omega$ and $t \in R_{s}(E)$ for some sufficiently small $s>0$, that is, $\Omega_{s} \cap G / B_{E} \subset \bar{X}_{\lambda}$. Since $\Omega_{s}=\bigcup_{F \subset E \subset \Delta}\left(\Omega_{s} \cap G / B_{E}\right)$ we have $\Omega_{s} \subset \bar{X}_{\lambda}$ for sufficiently small $s$. 
TheOREM 2. Let $\lambda \in a_{c}^{*}$ and assume $\operatorname{Re} \lambda \in a_{+}^{*}$. Then the Poisson transformation $\overline{\mathfrak{p}}_{\lambda}$ maps $C(K / M)$ into $C\left(\bar{X}_{\lambda}\right)$. In particular, if $\lambda \in \mathfrak{a}_{+}^{*}$, then $\bar{p}_{\lambda}(C(K / M)) \subset C(\bar{X})$.

Proof. Fix $F \subset \Delta$ and let $x \in \bar{X}_{\lambda} \cap G / B_{F}$. Let $g_{0} \in G$ be such that $x=\pi\left(g_{0}, t_{0}\right)$, where $t_{0 j}=1$ if $\alpha_{j} \in F$ and $t_{0 j}=0$ otherwise. Let $\varepsilon>0$ and choose a compact neighborhood $\Omega$ of $g_{0}$ in $G$ such that $\phi_{\lambda}^{F}(g) \neq 0$ and $\left|\mathfrak{p}_{\lambda}^{F} f(g)-\mathfrak{p}_{\lambda}^{F} f\left(g_{0}\right)\right|<\varepsilon / 2$ for all $g \in \Omega$. This is possible because of the continuity of $\mathscr{P}_{\lambda}^{F} f$ and $\phi_{\lambda}^{F}$.

Let $R_{s}, R_{s}(E)$ and $\Omega_{s}$ for $s>0$ and $E \supset F$ be as in the proof of Lemma 1. From Corollary 1 it follows that

$$
a_{t}^{\rho-\lambda} \mathscr{P}_{\lambda}^{E} f\left(g a_{t}\right) \rightarrow c_{\lambda}^{F}(E) \mathscr{P}_{\lambda}^{F} f(g)
$$

as $t \in R_{s}(E)$ and $s \rightarrow 0$, and the convergence is uniform in $g \in \Omega$. Combining this with (2.3) gives that $\mathfrak{p}_{\lambda}^{E} f\left(g a_{t}\right) \rightarrow \mathfrak{p}_{\lambda}^{F}(g)$, and the theorem follows.

For $\lambda=\rho$ this result is Proposition 4.2 in Korányi [9]. For $G=\operatorname{SL}(2, \mathbf{R})$ and $\lambda=\rho$ it is a classical theorem due to H. A. Schwarz (see [2, Theorem 4.20]).

REMARK. If $n=1$ and $\lambda=0$ the conclusion of Theorem 2 also holds (cf. Michelson [12, Theorem 1.3(i)]).

3. Fatou theorems. We will now, in an a.e. sense, extend to $L^{p}$-functions on $K / M$ $(1 \leqslant p \leqslant \infty)$ the convergence result of Theorem 1 . We consider the following types of convergence. Let $F \subset \Delta, \lambda \in \mathfrak{a}_{c}^{*}$ and $g \in G$.

Admissible convergence. We say that $a^{\rho-\lambda} \mathscr{P}_{\lambda} f(g a)$ converges to $c_{\lambda}^{F} \mathscr{P}_{\lambda}^{F} f(g)$ admissibly if for all compact sets $U \subset \bar{N}_{F}$ and $V \subset M_{F}$

$$
a^{\rho-\lambda} \mathscr{P}_{\lambda} f(g a \bar{n} m) \rightarrow c_{\lambda}^{F} \mathscr{P}_{\lambda}^{F} f(g m)
$$

as $a \underset{F}{\rightarrow} \infty$, uniformly for $\bar{n} \in U$ and $m \in V$.

Restricted admissible convergence. We say that $a^{\rho-\lambda} \mathscr{P}_{\lambda} f(g a)$ converges to $c_{\lambda}^{F} \mathscr{P}_{\lambda}^{F} f(g)$ restrictedly admissibly if for all compact sets $U \subset \bar{N}_{F}$ and $V \subset M_{F}$, and each $H \in \mathfrak{a}_{F}^{+}$,

$$
h_{t}^{\rho-\lambda} \mathscr{P}_{\lambda} f\left(g h_{t} \bar{n} m\right) \rightarrow c_{\lambda}^{F} \mathscr{P}_{\lambda}^{F} f(g m)
$$

as $t \rightarrow \infty$, uniformly for $\bar{n} \in U$ and $m \in V$. Here $h_{t}=\exp t H$.

Notice that if $f$ is continuous it follows from Theorem 1 that $a^{\rho-\lambda} \mathscr{P}_{\lambda} f(g a)$ converges to $c_{\lambda}^{F} \mathscr{P}_{\lambda}^{F} f(g)$ admissibly, because the set $\left\{a \bar{n} m a^{-1} \mid a \in A_{F}^{+}, \bar{n} \in U, m \in\right.$ $V\}$ is compact.

THEOREM 3. Let $1 \leqslant p \leqslant \infty$ and $f \in L^{p}(K / M)$. Assume that $\operatorname{Re} \lambda \in \mathfrak{a}_{+}^{*}$.

(i) There exists $p_{0}<\infty$ such that if $p>p_{0}$, then $a^{\rho-\lambda} \mathscr{P}_{\lambda} f(g a)$ converges to $c_{\lambda}^{F} \mathscr{P}_{\lambda}^{F} f(g)$ admissibly for almost all $g \in G$.

(ii) For any $p$ we have that $a^{\rho-\lambda} \mathscr{P}_{\lambda} f(g a)$ converges to $c_{\lambda}^{F \mathscr{P}_{\lambda}^{F}} f(g)$ restrictedly admissibly for almost all $g \in G$.

Proof. The proof is a simple generalization of the maximal function estimates given by Korányi and Stein for the case $\lambda=\rho$.

Let $L\left(\bar{N}_{F}\right)$ denote the space of measurable functions on $\bar{N}_{F}$, and let $\mathscr{M}$ be an operator (not necessarily linear) from $L^{p}\left(\bar{N}_{F} \times M_{F} \cap K\right)$ to $L\left(\bar{N}_{F}\right)$. Let $H \in a_{F}^{+}$. 
We say that $\mathscr{M}$ is an $H$-restricted maximal operator with respect to $p$ and $\lambda$ if it has the following properties:

$$
\left|a^{\rho-\lambda} \mathscr{P}_{\lambda} \Phi(\bar{n} a)\right| \leqslant \mathscr{M}(\Phi)(\bar{n})
$$

for all $\bar{n} \in \bar{N}_{F}, a=\exp t H(t \in \mathbf{R})$ and $\Phi \in L^{p}\left(\bar{N}_{F} \times M_{F} \cap K\right)$, where

$$
\mathscr{P}_{\lambda} \Phi(g)=\int_{\bar{N}_{F}} \int_{M_{F} \cap K} \Phi\left(\bar{n}_{1}, k\right) e^{\left\langle-\lambda-\rho, H\left(g^{-1} \bar{n}_{1} k\right)\right\rangle} d \bar{n}_{1} d k
$$

If $\mathscr{M}$ is an $H$-restricted maximal operator for each $H \in \mathfrak{a}_{F}^{+}$(i.e., (3.1) holds for all $a \in A_{F}$ ) we call it a maximal operator.

Theorem 3 follows once we have proved the following two propositions:

Proposition 1. Let $\lambda \in a_{c}^{*}$ such that $\operatorname{Re} \lambda \in a_{+}^{*}$.

(i) There exists $p_{0}<\infty$ and a maximal operator with respect to $p_{0}$ and $\lambda$ which is of weak type $\left(p_{0}, p_{0}\right)$.

(ii) For each $H \in \mathfrak{a}_{F}^{+}$there exists an $H$-restricted maximal operator with respect to $p=1$ and $\lambda$ which is of weak type $(1,1)$.

Proposition 2. Let $1 \leqslant p \leqslant \infty, f \in L^{p}(K / M)$ and $\lambda$ as above. Suppose there exists a maximal operator (respectively for each $H \in \mathfrak{a}_{F}^{+}$an $H$-restricted maximal operator) with respect to $p$ and $\lambda$ which is of weak type $(p, p)$. Then $a^{\rho-\lambda} \mathscr{P}_{\lambda} f(g a)$ converges to $c_{\lambda}^{F} \mathscr{P}_{\lambda}^{F}(g)$ admissibly (resp. restrictedly admissibly) for a.a. $g \in G$.

(See Korányi [10, p. 357] for the definition of weak $(p, p)$.)

Proof of Proposition 1. (i) For $\lambda=\rho$ such an operator is constructed in Korányi [10, Proof of Theorem 3.4]. It is easily seen that this operator in fact satisfies (3.1) for all $\lambda \in a_{c}^{*}$ with $\operatorname{Re} \lambda \in a_{+}^{*}$.

(ii) Choose $\gamma>0$ such that $\lambda=\gamma \rho+\mu$ with $\operatorname{Re} \mu \in a_{+}^{*}$. Then since $\operatorname{Re}\langle\mu, H(\bar{n})\rangle \geqslant 0$ for all $\bar{n} \in \bar{N}_{F}$ (cf. [3, Chapter 4, Corollary 6.6]) we get that

$$
\left|e^{\left\langle-\lambda-\rho, H\left(a^{-1} \bar{n}^{-1} \bar{n}_{1} k a\right)\right\rangle}\right| \leqslant e^{\left\langle-\lambda-\rho, H\left(a^{-1} \bar{n}^{-1} \bar{n}_{1} k a\right)\right\rangle} .
$$

Hence we may assume $\lambda=\gamma \rho$ with $\gamma>0$. Then it is easily seen (cf. [16, §8]) that the kernel $\exp \langle-\lambda-\rho, H(\bar{n})\rangle$ on $\bar{N}_{F}$ satisfies (a), (b) and (c) of [16, Theorem 1], and the proposition follows.

ProOF of Proposition 2 (cf. [10, Proposition 3.3]). We will prove that $a^{\rho-\lambda} \mathscr{P}_{\lambda} f(g \bar{n} a)$ converges to $c_{\lambda}^{F} \mathscr{P}_{\lambda}^{F} f(g \bar{n})$ (restrictedly) admissibly for a.a. $\bar{n} \in \bar{N}_{F}$ for each $g \in G$. For this we may take $g=e$ because of the invariance of $\mathscr{P}_{\lambda}$.

Let $L$ be a compact neighborhood of the identity in $K$, contained in the set $\kappa\left(\bar{N}_{F} M_{F}\right)$. By the invariance of $\mathscr{P}_{\lambda}$ we may assume supp $f \subset L$. For continuous functions the convergence holds by Theorem 1 . Since $f$ can be approximated in $L^{p}$ with continuous functions we can thus reduce to the case where $\|f\|_{p}$ is small. Then it suffices to prove the following two estimates for each $\varepsilon>0$ :

$$
\operatorname{mes}\left\{\bar{n} \in \bar{N}_{F}\left|\sup _{m \in V}\right| \mathscr{P}_{\lambda}^{F} f(\bar{n} m) \mid>\varepsilon\right\}<C_{1} \varepsilon^{-1}\|f\|_{p}
$$


(3.3) $\operatorname{mes}\left\{\bar{n} \in \bar{N}_{F}\left|\sup _{\bar{n}_{1} \in U, m \in V, a \in B}\right| a^{\rho-\lambda} \mathscr{P}_{\lambda} f\left(\bar{n} a \bar{n}_{1} m\right) \mid>\varepsilon\right\}<C_{2} \varepsilon^{-p}\|f\|_{p}^{p}$,

where $C_{1}$ and $C_{2}$ are constants and $B=A_{F}$ (respectively $B=\exp \mathbf{R} H$ ).

Notice first that from the following two estimates it follows that we may assume $U=V=\{e\}, f \geqslant 0$ and $\lambda$ real:

$$
\begin{gathered}
\left|\mathscr{P}_{\lambda}^{F} f(\bar{n} m)\right| \leqslant \int_{M_{F} \cap K}\left|f_{\lambda}(\bar{n} k)\right| d k \sup \left\{\left|e^{\left\langle-\lambda-\rho, H\left(m^{-1} k\right)\right\rangle}\right| \mid m \in V, k \in M_{F} \cap K\right\}, \\
\left|\mathscr{P}_{\lambda} f\left(\bar{n} a \bar{n}_{1} m\right)\right| \leqslant \int_{K}\left|f_{\lambda}(\bar{n} a k)\right| d k \sup \left\{\left|e^{\left\langle-\lambda-\rho, H\left(m^{-1} \bar{n}_{1}^{-1} k\right)\right\rangle}\right| \mid \bar{n}_{1} \in U, m \in V, k \in K\right\} .
\end{gathered}
$$

Now

$$
\left|\mathscr{P}_{\lambda}^{F} f(\bar{n})\right|=\int_{M_{F} \cap K} f(\kappa(\bar{n} k)) e^{\langle\lambda-\rho, H(\bar{n} k)\rangle} d k
$$

from which it follows that the left side of (3.2) is dominated by

$$
\begin{aligned}
& \varepsilon^{-1} \int_{\bar{N}_{F}} \int_{M_{F} \cap K} f(\kappa(\bar{n} k)) e^{\langle\lambda-\rho, H(\bar{n} k)\rangle} d k d \bar{n} \\
& \leqslant C_{1} \varepsilon^{-1} \int_{\bar{N}_{F}} \int_{M_{F} \cap K} f(\kappa(\bar{n} k)) e^{\langle-2 \rho, H(\bar{n} k)\rangle} d k d n=C_{1} \varepsilon^{-1}\|f\|_{1},
\end{aligned}
$$

where $C_{1}=\sup \left\{e^{\langle\lambda+\rho, H(\bar{n} k)\rangle} \mid \bar{n} \in \bar{N}_{F}, k \in M_{F} \cap K, \kappa(\bar{n} k) \in L\right\}$. Since $\|f\|_{1} \leqslant$ $\|f\|_{p}$, (3.2) follows.

Let $\Phi(\bar{n}, k)=f_{\lambda}(\bar{n} k)$ for $\bar{n} \in \bar{N}_{F}, k \in M_{F} \cap K$. Then we have $\mathscr{P}_{\lambda} f(g)=\mathscr{P}_{\lambda} \Phi(g)$ for $g \in G$, and (3.3) follows from (3.1) and the weak ( $p, p)$ property of $\mathscr{M}$.

This completes the proof of Theorem 3 .

From Theorem 3 we get the corresponding convergence result for $\mathfrak{p}_{\lambda} f$ by division with the spherical function (for simplicity stated only for real $\lambda$ ):

Corollary 2. Let $1 \leqslant p \leqslant \infty$ and $f \in L^{p}(K / M)$. Assume that $\lambda \in a_{+}^{*}$.

(i) There exists $p_{0}<\infty$ such that if $p>p_{0}$, then $\mathfrak{p}_{\lambda} f(g a)$ converges to $\mathfrak{p}_{\lambda}^{F}(g)$ admissibly for almost all $g \in G$.

(ii) For any $p$ we have that $\mathfrak{p}_{\lambda} f(g a)$ converges to $\mathfrak{p}_{\lambda}^{F}(g)$ restrictedly admissibly for almost all $\mathrm{g} \in G$.

For $F=\varnothing$ the admissible convergence a.e. was proved in Michelson [12] for $f \in L^{\infty}$ and the restricted admissible convergence a.e. for $f \in L^{1}$ in Sjögren [15]. For $\lambda=\rho$ and $F$ arbitrary they were proved in Korányi $[9,10]$ and Stein [16].

REMARK. The condition on $\lambda$ that $\operatorname{Re} \lambda \in a_{+}^{*}$ in Theorems 1 and 3, Proposition 2 and Corollary 2 can be weakened slightly to $\operatorname{Re}\langle\lambda, \alpha\rangle>0$ for all $\alpha \in \Sigma^{+}$with nonzero restriction to $\mathfrak{a}_{F}$. This follows easily from the proofs given.

NOTE ADDED IN PROOF. In a recent preprint, Admissible convergence of Poisson integrals in symmetric spaces (Chalmers University of Technology, the University of Göteborg, 1985), P. Sjögren obtains admissible convergence for $f$ in $L^{p}$ with any $p>1$ (that is, $p_{0}=1$ in Theorem 3(i)). 


\section{REFERENCES}

1. S. Helgason, $A$ duality for symmetric spaces with applications to group representations, Adv. in Math. 5 (1970), 1-154.

2. __ Topics in harmonic analysis on homogeneous spaces, Progress in Math., Vol. 13, Birkhäuser, Boston, Basel and Stuttgart, 1981.

3. __ Groups and geometric analysis. Integral geometry, invariant differential operators and spherical functions, Academic Press, Orlando, Fla., 1984.

4. S. Helgason and A. Korányi, A Fatou-type theorem for harmonic functions on symmetric spaces, Bull. Amer. Math. Soc. 74 (1968), 258-263.

5. M. Kashiwara, A. Kowata, K. Minemura, K. Okamoto, T. Oshima and M. Tanaka, Eigenfunctions of invariant differential operators on a symmetric space, Ann. of Math. (2) 107 (1978), 1-39.

6. A. W. Knapp, Fatou's theorem for symmetric spaces. I, Ann. of Math. (2) 88 (1968), 106-127.

7. A. W. Knapp and R. E. Williamson, Poisson integrals and semisimple groups, J. Analyse Math. 24 (1971), 53-76.

8. A. Koranyi, Boundary behaviour of Poisson integrals on symmetric spaces, Trans. Amer. Math. Soc. 140 (1969), 393-409.

9. __ Poisson integrals and boundary components of symmetric spaces, Invent. Math. 34 (1976), $19-35$.

10. Compactifications of symmetric spaces and harmonic functions, in Analyse Harmonique sur les Groupes de Lie. II, Séminaire Nancy-Strasbourg 1976-1978 (P. Eymard et al., eds.), Lecture Notes in Math., vol. 739, Springer-Verlag, Berlin and New York, 1979, pp. 341-366.

11. L.-Å. Lindahl, Fatou's theorem for symmetric spaces, Ark. Mat. 10 (1972), 33-47.

12. H. L. Michelson, Fatou theorems for eigenfunctions of the invariant differential operators on symmetric spaces, Trans. Amer. Math. Soc. 177 (1973), 257-274.

13. T. Oshima, A realization of Riemannian symmetric spaces, J. Math. Soc. Japan 30 (1978), 117-132.

14. H. Schlichtkrull, Hyperfunctions and harmonic analysis on symmetric spaces, Progress in Math., Vol. 49. Birkhäuser, Boston, Basel and Stuttgart, 1984.

15. P. Sjögren, $A$ Fatou theorem for eigenfunctions of the Laplace-Beltrami operator in a symmetric space, Duke Math. J. 51 (1984), 47-56.

16. E. M. Stein, Boundary behaviour of harmonic functions on symmetric spaces: Maximal estimates for Poisson integrals, Invent. Math. 74 (1983), 63-83.

17. D. Vogan, Representations of real reductive Lie groups, Progress in Math., Vol. 15, Birkhäuser, Boston, Basel and Stuttgart, 1981.

School of Mathematics, The Institute for Advanced Study, Princeton, New Jersey 08540

DePartment of MAThematics, University of Copenhagen, UniversitetsParken 5, DK-2100 København Ø, Denmark (Current address) 